\title{
Young-onset Parkinson disease
}

INSERM

\section{Source}

INSERM. (1999). Orphanet: an online rare disease and orphan drug data base. Youngonset Parkinson disease. ORPHA:2828

Young-onset Parkinson disease (YOPD) is a form of Parkinson disease (PD), characterized by an age of onset between 21-45 years, rigidity, painful cramps followed by tremor, bradykinesia, dystonia, gait complaints and falls, and other non-motor symptoms. A slow disease progression and a more pronounced response to dopaminergic therapy are also observed in most YOPD forms. 